\title{
THE PAEDIATRIC SYNDROME OF TRAUMATIC MYELOPATHY WITHOUT DEMONSTRABLE VERTEBRAL INJURY *
}

\author{
By David J. E. Cheshire, M.B., B.S.(Lond), D.Phys.Med.(Eng), D.P.R.M.(Aust.) \\ Southwest Regional System for Treatment of Spinal Injury, Good Samaritan Hospital, \\ I033 E. McDowell Road, Phoenix, Arizona 85062, U.S.A.
}

Abstract. Three detailed case histories are added to those previously published in the literature and from the evidence now available a proposal is made that a paediatric syndrome of traumatic myelopathy without demonstrable vertebral injury be recognised as a definite clinical entity. The mechanisms of injury, evolution, patterns of paralysis and pathological evidence are discussed. The conclusion is drawn that no form of treatment yet known can influence the prognosis in this syndrome.

\section{Introduction}

As an arbitrary subdivision, spinal cord trauma in man may be described as occurring during birth, in the period between infancy and I 3 years, between I 3 years and middle age, and in persons of middle and old age. The first full description of intrapartum spinal cord injury was that of Parrot (1869) and since that paper there have been many other reports, some of quite large series. The classical mechanism would appear to be that of hyperextension of the cervical spine in a difficult breech delivery and, in these infants, the resulting tetraplegia has been described to be associated with 'usually normal' $\mathrm{X}$-rays of the spine (Byers, 1975). This excellent review paper indicates both the magnitude and complexity of the problem and this group of spinal injuries will not be further discussed in the present paper.

Spinal cord injuries in the age-group from infancy to 13 years may be open or closed and may or may not be associated with demonstrable skeletal injury. It is the closed injury in which no skeletal injury can be demonstrated which it is proposed to explore in detail in this paper.

In the age-group from I 4 to middle age, or arbitrarily 45 years, it is excessively rare to find closed spinal cord trauma in a patient in whom skeletal injury cannot be demonstrated even though the demonstration may be difficult and may require, in addition to the conventional radiographic techniques, the use of functional films, tomography, or myelography.

In patients of middle or old age there is now a clearly defined syndrome of closed spinal cord injury without demonstrable skeletal injury. This syndrome evolved from the first description by Crooks and Birkett (I944) of hyperextension injuries of the spinal cord in patients with spondylotic spines, through the classical paper of Taylor (I95I) who reported a case of cervical cord injury without autopsy evidence of traumatic damage to the cervical spine and then, through the pioneer work at the London Hospital, came the true understanding of the syndrome of cervical spondylosis (Brain et al., 1952; Wilkinson, I960; Brain and Wilkinson, 1967). The final link in the total delineation of this syndrome was the paper by

* This project was supported in part by U.S. Department of Health, Education and Welfare, Rehabilitation Services Administration Grant Number 13-P-55258/9. 
Schneider et al. (1954) in which the syndrome of acute central cervical spinal cord injury was described with special reference to the mechanism of hyperextension injuries of the cervical spine.

It is this knowledge of the gradual evolution of full comprehension of the syndrome of middle age which has led the author to attempt to assemble current knowledge of spinal cord trauma without demonstrable bony injury in the paediatric age-group and to hope that the paediatric syndrome will soon reach the same level of understanding as that which currently exists in the middle age-group.

When one attempts to study closed spinal cord trauma in children, one immediately comes up against the double difficulty of a very low incidence which has been estimated at, respectively, 3.3 per cent and 2.5 per cent of all spinal injuries (Forni, I947; Gelehrter, I957) and, very frequently, case reporting which is inadequate to permit an author to draw conclusions from the experience of others. Examples of the foregoing are the papers of Melzak (I969) who, on reviewing the 4470 patients with spinal cord lesions who had been admitted to the British National Spinal Injury Centre since 1944, reported the admission of 93 children, but of these, only 29 were due to traumatic lesions. Of these 29 children with traumatic lesions it was recorded that no fracture of the vertebral column could be detected in I6. Regrettably one cannot use this information further to delineate a paediatric syndrome. In comparable manner, Audic and Maury (I969) report 2I traumatic lesions of the spinal cord in children under I6, but say no more than that of the 2I of traumatic aetiology 'very often' no fracture was detected.

In attempting to delineate a paediatric syndrome of closed spinal trauma without demonstrable bony injury the author finds a very sparse literature. The first paper of real assistance is that of Burke (I97I), and this paper is particularly helpful to the present author as, at the time of writing that paper, he was the Director of the Spinal Injuries Center for Victoria, Australia, and the treating physician of the patients reviewed. However, no surgery was performed and all the children survived. There is thus no direct visual examination of the spinal cord against which to test one's clinical impressions concerning the aetiology of the lesions. Burke's I974 paper is of assistance in that it contributes some surgical and autopsy evidence but it does not include any reference to the mechanics of injury nor to the clinical neurological presentations of the two children with incomplete lesions out of a total of 24 patients. The paper of Glasauer and Cares (1972) contributes two good case histories with, in one, macroscopic examination of the cord at laminectomy and, in the other, macroscopic and microscopic examination at autopsy. The paper of Ahmann et al. (1975) is primarily concerned with the autopsy findings in two children, but there is some aetiological mechanistic information as well as clinical neurological information. The evidence available to the author includes that derived from three children who have recently been under his personal care, but, although the study of these children contributes a great deal in terms of the biomechanics of injury and clinical neurological presentation, it does not include any of the histological evidence which is necessary fully to understand the syndrome.

\section{Case Reports}

328 cases of acute spinal cord trauma were treated in the Southwest Regional System between I June 1970 and 3I December 1975. Of these, four were below the age of $I 3$ years, an incidence of $I \cdot 2$ per cent, and three presented the syndrome to be described. 
Patient No. I. D. Y., a $6 \frac{1}{2}$-year-old girl, tried to turn a backward somersault off her bed, but landed on her head and, following the impact of the head, her body fell to the floor in a position of extreme hyperextension of the spine. There were no eye-witnesses, but repeated interrogation of the child has convinced the author of the veracity of the described mechanism. She experienced immediate severe low dorsal pain and when she tried to get to her feet she was only able to get to a kneeling position on her hands and knees. This is taken to imply her retention of voluntary control of the hip muscles immediately after the accident. The girl called for her father, who tried to help her stand, but she was unable to do so. He picked her up, laid her on the bed and on examination a few minutes later found her wholly unable to move her legs. She was taken to a neurosurgical centre where she was found to have a complete motor and sensory flaccid paraplegia below the level of the I I th dorsal segment. Plain X-rays, myelography and spinal angiography were performed, but all were normal. No surgery was performed.

The child's clinical condition remained unchanged for 5 months until she began to pass urine in a reflex manner and at this time clinical examination showed there to be a return of the sacral reflexes.

The stabilised neurological state 3 years after injury is that of a complete motor and sensory paralysis below D I I with persistent flaccidity of the legs and arreflexia down to and including SI. Reflex function has returned in the S2-5 segments. Serial follow-up spinal X-rays are all negative for evidence of skeletal injury.

It is felt that this child sustained a hyperextension injury and, with no demonstrable skeletal injury, a complete paraplegia below the level of D I I which, whilst incomplete for the first few minutes, became complete within minutes. The child has remained with a lesion which has remained flaccid from DI2 through SI with a return of spinal cord reflex function in the sacral segments. In this child, it is tempting to postulate a fulcrum at about the IIth or I2th dorsal vertebrae with a resulting longitudinal lesion of the cord from DI2 through SI and an intact conus. The aetiology must remain one of speculation with both traction and infarction being possibly implicated, but which mechanism and in what proportion is absolutely unknown.

Patient No. 2. E. Y., a boy of 3 years and 3 months, was rolling and playing with his father when he tripped and fell, landing on his cervico-dorsal junction forcing an extreme hyperflexion thrust. The boy immediately went limp, turned pale and apparently unresponsive. His parents revived him with cold water and took him to the Emergency Room of the nearest hospital, where the examining doctor could find no abnormality and suggested a possible diagnosis of a seizure. The child was taken home, put to bed, and slept for several hours. On waking he complained of pain in the neck, had difficulty in standing because of weakness of the legs and had difficulty in the movement of his upper limbs, especially in the use of his hands for the manipulation of small objects. The parents took him back to hospital where, about 5 hours after injury, he was examined by a pediatrician who found weakness of the muscles of the shoulder girdle bilaterally, weakness of the flexors and extensors of the elbow, and no demonstrable movement of the fingers. No motor loss was demonstrated in the trunk or legs. Examination of reflex function is recorded as showing diminution of the biceps, brachioradialis and triceps jerks. Sensory examination is recorded as showing touch and pin prick to be equal and normal bilaterally throughout the entire body.

The child was admitted to hospital and the following morning, about I8 hours after injury, he was seen by a neurosurgeon who noted that there was flaccid paralysis of the upper limbs and a spastic paresis of the lower limbs. Twenty-four hours later, or about 42 hours after his injury, the neurosurgeon recorded that the legs were weaker than they had been on the previous day and that intercostal muscle function had become impaired. At this time standard X-rays of the spine, functional films and myelography were performed and all were normal.

The boy was first seen by the author about 48 hours after injury and examination at 
this time showed normal function of the cervical muscles bilaterally but complete loss of all voluntary motor function from the deltoids, through both arms and the whole trunk. With persistence, it was possible to demonstrate voluntary motor function in all muscles of the thigh bilaterally, but despite repeated efforts, it was not possible to demonstrate voluntary function in any muscles below the knee save for the extensor hallucis longis and the gastrocnemius bilaterally. The tone of the upper limbs was diminished and that of the lower limbs significantly increased. The tendon reflexes of both upper limbs were absent and the superficial abdominal reflexes were also absent. The knee jerks and ankle jerks were exaggerated bilaterally and the plantar responses were extensor and associated with withdrawal at the hip. Anal tone was good and the bulvocavernous reflex was positive. Sensory examination was difficult in this young child, but it was the author's opinion that light touch and pin prick were almost, if not quite, unimpaired throughout the body and symmetrical on the two sides. The author was not able to estimate with any accuracy whether or not the modalities of posterior column sensory function were impaired.

A diagnosis was made of an incomplete spinal cord injury below the level or $\mathrm{C}_{4}$, of acute central cervical spinal cord injury type, due to a hyperflexion injury, but without any evidence of skeletal injury.

In view of the proposed aetiology, it was felt to be of particular importance to evaluate posterior column sensory function and on examination the following day the author felt reasonably certain that the child did not appreciate the difference between the vibrating tuning fork and the touch of a non-vibrating tuning fork.

This boy's recovery was rapid. On the fourth post-accident day there was evidence of improvement in the function of the intercostal muscles and return of function in the anterior and posterior trunk muscles. Also, on the same day there was evidence of a return of voluntary motor function in the long flexors and extensors of the toes on both sides. By the tenth day, there was unequivocal return of voluntary motor function in all muscles throughout the body and there was evidence of both a return of tone in the upper limbs and a diminution in the hypertonicity of the lower limbs.

At 3 weeks from injury, the child had regained virtually normal voluntary motor function throughout the body except for the right forearm and hand. There was at this time significant weakness of the hand muscles. In the upper limbs there was still relative hypotonia with the tendon jerks present, but diminished, and in the lower limbs relative hypertonia with exaggeration of the tendon jerks and extensor plantar responses. Sensory examination showed light touch and pain to be normal throughout the body and by this time there was certainty that both vibration sense and joint position sense were present throughout the body.

The child was followed closely and at 12 months from injury he was absolutely normal in all neurological functions in the left arm, the trunk, and both lower limbs. Bladder and bowel function was also normal. The area in which there was not full return of normal function was the right upper limb. Here there was normal function of the muscles of the shoulder girdle and of the elbow flexors, but a persistent triceps weakness and a persistent slightly spastic paresis of both the extrinsic and intrinsic muscles of the hand. Sensation in this limb was normal. Although there was both an academic and a functional impairment in the right upper limb, the boy, who was naturally right handed, continued to use his right hand for all functional activities.

Factors which are felt to be of importance in the evaluation of this child are the unequivocal hyperflexion injury and the slow evolution to the maximum degree of paralysis which was that of an incomplete tetraplegia below $\mathrm{C}_{4}$ presenting as an acute central cord syndrome. The child made a rapid recovery, but at 2 months still had the residua of a spastic paresis of the extrinsic and intrinsic muscles of the right hand. If one postulates a hyperflexion traction injury of the cord it is interesting to note the apparent early complete loss of posterior column function, but the recovery of this modality to a degree greater than was achieved in at least one area 
of the more ventrally placed lateral cortico-spinal tract. Clearly one cannot postulate anatomical disruption of the cord; a degree of infarction is possible, but improbable. Contusion would appear to be most likely. Again, however, the aetiology remains speculative.

Patient No. 3. T. M., a boy aged 2 years and 2 months, was playing in the garden when, at about 9.00 p.m., he was observed by both parents to run with some force into collision with a metal table, striking his right cheek hard against the table edge. He fell backwards striking the back of his head on the ground, but did not lose consciousness. He cried and was immediately carried to bed by his father, who recollects that the overall feel of the boy's body appeared normal. As the father left the room, he observed the boy to be grasping his sister's arm with his hand and to be standing apparently without difficulty at the side of the bed. The following morning when the father went to get him from bed the boy was unable to sit up and it became apparent that he was unable to move his limbs. The father then picked him up and immediately noted that the whole body felt 'floppy', a feeling which he clearly differentiates from the feel of the body when he carried the boy to bed on the previous evening.

The boy was taken to hospital and seen by a neurosurgeon. At the first examination there was evidence of an abrasion and some swelling over the right zygomatic arch, but no other evidence of head injury. There was complete absence of voluntary motor function in the upper limbs, trunk and lower limbs. The upper limbs were flaccid and arreflexic, but in both legs there was flexor withdrawal to light stimulation and an extensor plantar response. Sensory examination showed there to be complete loss of all sensation below the level of $\mathrm{C}_{5}$. Routine X-rays of the cervical spine, functional films and a myelogram were all normal. No surgery was performed. The boy was first seen by the author on the eighth day when there was noted to be normal voluntary power in all muscles of the neck but complete absence of all voluntary motor function in upper limbs, trunk and lower limbs. The upper limbs were hypotonic, but the legs were hypertonic. Examination of reflex function showed that all tendon jerks were absent in the upper limbs, and the superficial abdominal reflexes were absent. In the lower limbs, the knee and ankle jerks were exaggerated and the plantar responses were extensor and accompanied by a flexion-adduction pattern of withdrawal. There was complete absence of all sensory modalities below $\mathrm{C}_{4}$. A pattern of reflex micturition had already become established, with residuals as low as 5 mills.

The impression on first examination was that this boy had sustained a motor and sensory complete tetraplegia below the level of $\mathrm{C}_{4}$ due to a hyperextension injury of the cervical spine, but without evidence of skeletal injury.

On the fourteenth day after injury voluntary motion was seen in the left deltoid and pectoral muscles. On the seventeenth day, voluntary motion was seen in the left biceps brachii, and there was evidence of voluntary motion in the intercostal, abdominal and hip flexor muscles. There was gradual improvement in the muscles of both upper limbs down to the extensor carpi radialis, but no voluntary function was seen in the triceps or in the extrinsic or intrinsic muscles of the hands. There was progressive improvement in the muscles of the trunk and of the lower limbs. The upper limbs remained flaccid and arreflexic and the lower limbs became more spastic and hyperreflexic. There was also evidence of return of sensory function in the 5th and 6th cervical segments and in the trunk and lower limbs.

Evaluation 7 months after injury showed that the pattern of the upper limbs was unchanged with voluntary motor function extending only down to extensor carpi radialis. There was progressive improvement in the power of the muscles of the trunk and lower limbs. The right upper limb remained arreflexic, but tendon reflexes had returned in the left upper limb. The reflexes of the lower limbs remained exaggerated and the plantar responses extensor.

At I year there was still no return of extrinsic or intrinsic hand function, but the lower limbs, although spastic and paretic, had improved to a stage which permitted early ambulation training. At 18 months there was little change with the right upper limb 
remaining arreflexic, but the tendon jerks in the left upper limb were now exaggerated and the Hoffmann reflex was positive. The lower limbs remained spastic and paraparetic, but there was further improvement in limited ambulation.

It is felt that this boy sustained an incomplete cervical spinal paralysis below the level of $\mathrm{C}_{4}$ of the pattern characteristic of an acute central spinal cord syndrome. The only variant from the classical central spinal cord syndrome is the persistence of arreflexia in the right upper limb. It will be recalled that the primary injury was one of impact of the right zygomatic arch against the edge of a metal table and one must postulate a rotation component resulting in a lower motor neuron lesion at the site of injury.

\section{Review of the Literature}

The literature to review is remarkable only in its scarcity. Burke (I97I) in his review of spinal cord trauma in children describes seven cases. Of these, four have normal X-rays and are appropriate for inclusion in this paper. A fifth patient described by Burke as having normal X-rays 'except for separation of the spinous processes between $\mathrm{D}_{3}$ and 4' must be excluded because it is felt that the separation of the spinous processes is an unequivocal demonstration of abnormality of X-ray appearance and hence must exclude the patient from consideration. Of Burke's four cases, two are of the greatest interest in that they are two little girls, aged respectively 15 and 18 months, who sustained the same mechanism of injury, namely, hyperflexion of the spine by a slow compression between the ground on which they were crawling and the slow-moving wheel of the vehicle that their father was garaging. These children sustained, respectively, paraplegias complete below the level of the fourth dorsal segment and the third dorsal segment and both remained totally flaccid and arreflexic below the level of injury. A further item of historical interest is that in the 18 -month-old girl there is some evidence, though of uncertain reliability, that she was not immediately paralysed, but the paralysis developed during the hours following injury during which she was sleeping. Burke's two other patients were males, of respectively 6 and 8 years, the first of whom was knocked over in the street by a speeding car and the second was a passenger in his father's car who was hyperflexed under the dashboard of the car in a collision. These boys developed complete spastic paraplegias, the first asymmetric below $\mathrm{D}_{2}$ on the left side and $\mathrm{D}_{4}$ on the right, and the second below $\mathrm{D}_{9}$. In both these boys there is the strongest historical evidence that the onset of paraplegia was immediate. All four children have survived, hence there is no evidence to assist in the elucidation of the aetiology of the spinal cord injury.

Burke (1974) published the results of a review of the charts of spinal cord injuries sustained in children treated at Rancho Los Amigos Hospital in California. Of the post-birth injuries, there were 22 complete lesions and two incomplete lesions. There is no information in this paper concerning the time relationship between injury and paralysis nor of the clinical neurological pattern of the children with incomplete lesions. One of the 24 children died, a child of I I months with a complete tetraplegia below the 5 th cervical segment who died $3 \frac{1}{2}$ months after injury. An autopsy of this child showed that the cord had 'a constricted area (compressed) for four $\mathrm{cm}$ above the cervical enlargment'. This finding will be discussed later. There is a report of the findings at laminectomy in a second case.

Glasauer and Cares (1972) provide details of two infants who sustained complete spinal cord injuries of immediate onset, both remaining complete. In 
one there is visual evidence obtained at laminectomy, and, in the other, autopsy evidence.

Ahmann et al. (1975) describe two patients, the first of whom was a 4-year-old girl who, on returning home from a playground, complained of back pain and said that she had been pushed out of a swing. It was not known if she had fallen, but she was walking when she returned home. She awakened from a nap several hours later and fell on getting out of bed. She again complained of back pain, was unsteady when she walked and her arms appeared weak and limp. On physical examination, she had complete flaccid paralysis of the upper limbs, but retained voluntary motion and normal reflexes in the lower limbs. Twelve hours after admission to hospital, she had deteriorated with the lower extremities now being flaccid and with retention only of minimal corsi- and plantar-flexion of the feet. She was totally arreflexic. The details of neurological progress are sparse, but the child died on the forty-eighth hospital day and the autopsy findings will be described below. The second case reported by these authors is that of a 22-month-old boy who received minor trauma to the left jaw and face from cuffing. He fell to the floor, but immediately regained a standing posture. Although he could walk, he was noted to have difficulty holding a glass of water with his right hand. Over the next four hours he became tetraparetic and developed respiratory difficulty necessitating intubation. Six hours after the trauma he was admitted to hospital at which time he had a flaccid tetraplegia below $\mathrm{C}_{4}$. Again the clinical neurological details are scanty, but the child died 6 months after injury and he also came to autopsy.

Thus I3 cases are available for further detailed analysis, both of the clinical syndrome and of such pathological evidence as exists (Table I). Of these three are the children reported in this paper in whom there is, in two, certain evidence of a hyperextension injury and, in one, equally certain evidence of a hyperflexion injury. One of the children remained complete, the other two became incomplete. In all three children there is the strongest evidence of the evolution of paralysis rather than immediate onset. In none of these children is there any pathological information. Of the four children from Burke's I97I paper, there is possible evidence of a delayed onset in one, no information in one and certain evidence of immediate onset in two. In none of the children is there any pathological evidence, but in all four there are grounds for speculation concerning the aetiology of the paralysis. Burke's 1974 paper provides evidence from laminectomy in one child and from autopsy in another. Glasauer and Cares (1972) provide clinical information in two patients with visual examination of the cord in both. Ahmann et al. (1975) provide a very clear history of the gradual evolution of tetraplegia in two children. The authors ascribe the spinal cord injury to a hyperextension mechanism in both children, but this is felt to be presumptive rather than proved. These two cases are of particular interest in that they provide the best evidence of aetiology to be derived from autopsy examination. This evidence is extremely interesting and persuasive and will be discussed below. It is, however, unfortunate that the autopsy reports are exclusively concerned with the spinal cord and do not give any information concerning the spine or of the surrounding soft tissues.

\section{Pathology}

In discussing the aetiology of the paediatric syndrome, it is felt that it is first necessary to amend the opinion expressed by Burke (I97I) which was, until recently shared by the present author, that this is a syndrome essentially associated 
PAPERS READ AT THE ANNUAL SCIENTIFIC MEETING, I 976

㲖

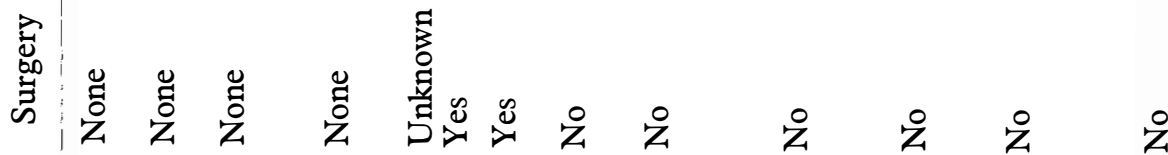

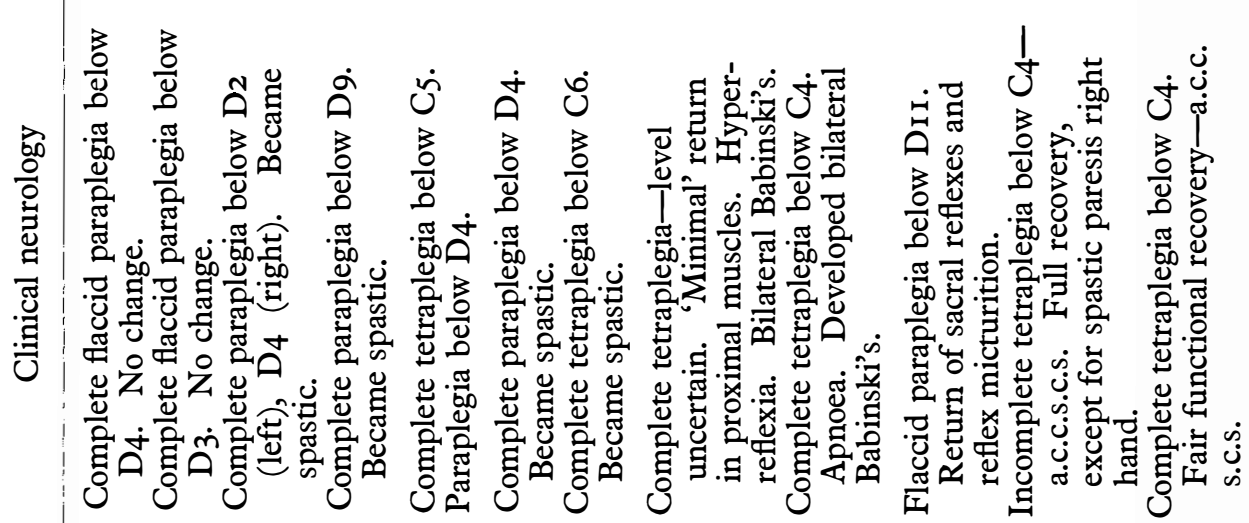

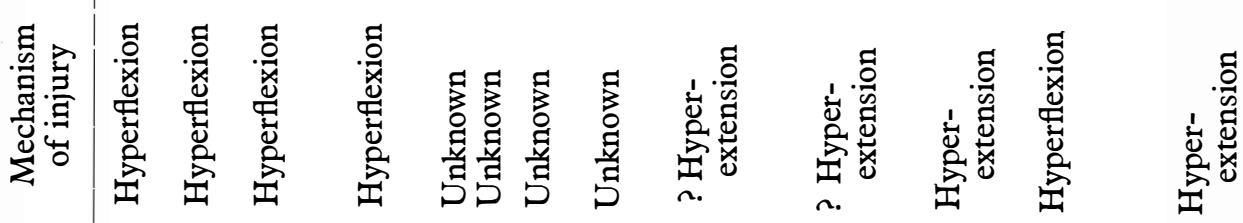

|

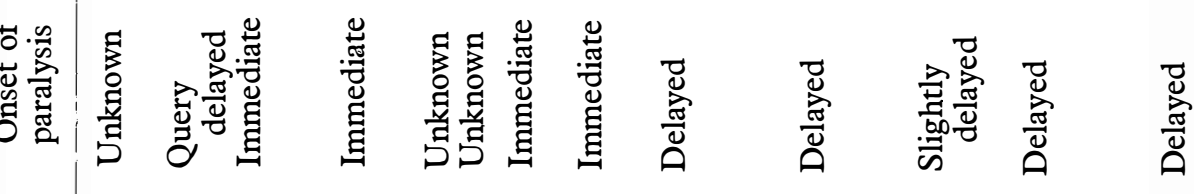

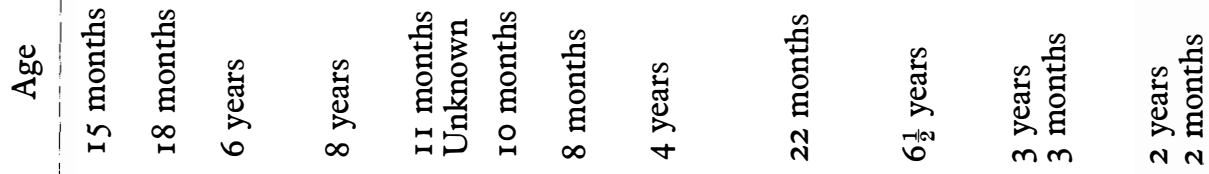

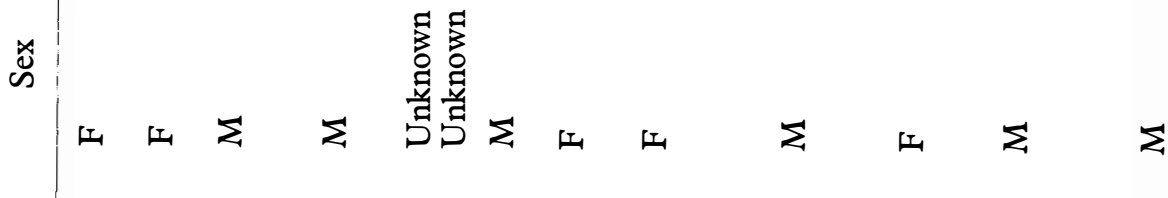

旁:

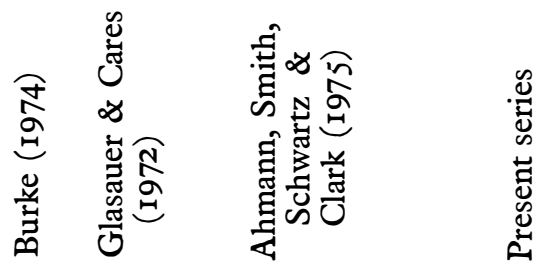


with hyperflexion injuries. In the light of the histories of two of the children reported in this paper in which there is unequivocal evidence of a hyperextension injury, and the less definite but inferential evidence of hyperextension in the aetiology of the patients reported by Ahmann et al. (1975), it now appears clear that either hyperflexion or hyperextension may be the aetiologic mechanism.

To investigate the pathology it is necessary to study the gross macroscopic evidence obtained at both laminectomy and autopsy and also the histological evidence obtained at autopsy. It is also possible to use the evidence of clinical neurological presentation and evolution as a basis for exploring inferential pathological mechanisms.

Burke (I974) reports the macroscopic evidence at autopsy of 'a constricted area (compressed) for four $\mathrm{cm}$ above the cervical enlargement', and argues that a 'constricted' segment of spinal cord of $4 \mathrm{~cm}$ in length above the cervical enlargement in an I I-month-old child is much more likely to be a stretching than a compressing lesion. He also records the operative findings of a traumatised spinal cord six segments below the level of clinical neurological section and suggests that this represents a long segment of damaged cord. He also reports having seen gross attenuation of the cord at autopsy in an adult and Bedbrook (1966) mentions similar findings. It is felt that this autopsy evidence sustains the hypothesis evolved by Burke and the present author that longitudinal stretching is likely to be a major force in the pathogenesis of spinal cord lesions in children which are not associated with evidence of skeletal injury. It is postulated that the elastic cartilaginous spine of a young child may be grossly distorted by deforming forces, but that the spinal column does not disrupt through its inherent elasticity. However, the spinal cord and the meninges are unable to withstand this degree of traction and, usually, torsion, and the neural tissue disrupts longitudinally. A mechanism is postulated which is entirely compatible with the evidence, both macroscopic and microscopic, which has been described by Bedbrook (I966) in adults. This postulated mechanism is compatible with, and strengthened by, Leventhal's (1960) experiments with autopsy specimens in which he demonstrated that the spine of the neonate can be stretched 2 inches whilst the cervical spinal cord can be pulled down only onequarter of an inch before rupturing. It is also possible to use the hypothesis of a longitudinal traction injury mechanism to explain the permanently flaccid lesions below $\mathrm{D}_{4}$ and $\mathrm{D}_{3}$ in the two children described by Burke (I97I) and the lesion of Patient No. I in the present series.

This is, however, unlikely, both in hypothesis and in evidence, to be the only pathological mechanism. Wolman (1964) suggested a momentary nipping of the cord when vertebrae are displaced, or momentary stretching of the cord over the posterior aspect of the vertebral bodies with forcible flexion. Whatever the exact details, it is persuasive to postulate a transection-type injury which is very much in accord with the clinical histories and visualisation of the cords in Glasauer and Cares' (1972) cases and also in Burke's (I97I) two boys who showed the immediate onset of a complete transverse spinal cord injury syndrome. Glasauer and Cares (1972) report the observation at laminectomy of segmental atrophy of the cord at the level of injury and approximately two-segment level atrophy at autopsy in their other case. In this case massive central necrosis was seen on microscopy.

Additional evidence concerning the microscopic anatomy of the spinal cord injury in this syndrome is provided in the paper of Ahmann et al. (1975). In the first case serial sectioning of the spinal cord demonstrated ischaemic necrosis beginning at $\mathrm{C}_{3}$ and extending to $\mathrm{DI}_{1}$. The infarction involved the medial lateral grey columns, the lateral part of the anterior horn and the base of the posterior grey 
column, sparing the medial tip of the anterior horn and the commissure. The infarction was maximal at $\mathrm{C}_{4}$. Unfortunately, it is difficult to make an accurate comparison between the observed microscopic findings and the clinical neurology as the details of the latter are deficient. The second case describes the autopsy appearances of a spinal cord which was markedly flattened and distorted from $\mathrm{CI}_{\mathrm{I}}$ through C5. Microscopic sections of the cervical cord and brain stem demonstrate ischaemic necrosis with complete loss of cord substance from $\mathrm{CI}_{\mathrm{I}}$ through $\mathrm{C}_{5}$. The ischaemic infarct also extended into the lower medulla to the level of the inferior olive.

Ahmann and his colleagues postulate a mechanism of transient vertebral artery occlusion followed by severe reduction of blood flow to the cord secondary to spasm. They feel that it is unlikely that the observed pattern of infarction resulted from occlusion of the anterior median spinal artery for the infarction pattern in anterior median spinal artery occlusion is generally much more extensive and they found no occlusion in the artery on either gross examination or microscopic section. They discuss the possibility that the infarction pattern might have been related to a vascular anomaly such as the absence of the segmental medullary artery to the lower cervical cord which occurs in Io per cent of individuals. The central part of the mid to lower cervical cord then would depend on the small segmental medullary artery supplying the upper thoracic cord. They also speculate that this infarction pattern might have resulted from infarction of the terminal arterial bed in the dorsal ventral plane. Such a watershed area has not been documented experimentally, but the area involved corresponds to the end-points of flow from the central sulcal area. Compromised flow in both anterior and posterior circulation might have created the infarction pattern seen. Essentially, however, these authors describe but do not explain an observed infarction pattern.

\section{Discussion}

From the foregoing, it is felt that it is possible to state with certainty that there is an entity which may appropriately be described as the paediatric syndrome of traumatic myelopathy without demonstrable vertebral injury. Although the number of cases which have been studied in fullest detail remains small, it may also be stated with certainty that this syndrome may be caused by either hyperflexion or hyperextension mechanisms.

From the cases studied, it is also apparent that the syndrome may present in two ways, that of profound paralysis occurring immediately after the trauma or as a progressive paralysis starting to be apparent from a few minutes to a few hours after injury and progressing to its maximum for as long as 48 hours.

In the majority of children the spinal paralysis is complete and this would appear to be particularly true in those children in whom the onset of the paralysis is immediate. There are, however, as exemplified by the two boys whose histories are published in this paper, a group of children in whom the lesion is incomplete and it is not felt to be coincidental than in both these children the onset of spinal paralysis was delayed.

In those children who develop incomplete spinal paralysis, the number of patients yet analysed in detail does not permit any conclusions to be drawn, but the classical presentation of the acute central cervical spinal cord syndrome (Schneider et al., 1954) has been demonstrated and, moreover, demonstrated in association with both hyperflexion and hyperextension injuries.

Evidence at surgery and at autopsy would at this present incomplete stage of 
understanding of the syndrome, suggest that three mechanisms may be involved. The first of these is longitudinal traction of the cord, the second, transverse section of the cord, and the third, infarction of the cord. It is tempting to suggest that there may be an association between the immediate onset of paralysis and a longitudinal traction injury or a transection injury, and between a delayed onset and an infarctive mechanism, but at the present time this can be no more than speculation.

In addition to the great importance of recognising the existance of this syndrome it would appear to be abundantly proved that surgery has nothing to offer in the treatment of these patients and, in fact, surgery can only be counterproductive in adding a potential skeletal instability to an existing spinal paralysis. It is also difficult in the present understanding of this condition to visualise any benefit to be derived from the administration of any pharmacological agent.

\section{Conclusion}

It is felt that a case has been made for the definition of a paediatric syndrome of traumatic myelopathy without demonstrable vertebral injury which may in many ways be likened to the syndrome which is seen in middle and old age. Although in the latter group of patients, the aetiological mechanisms are better understood, there still remains in both considerable uncertainty with regard to the micropathology of cord destruction. Both groups share the common problem of being inadequately understood and hence patients sustaining these injuries are liable to lack of recognition, delayed diagnosis and mistreatments. The answer to the better management of both groups is a higher index of suspicion in relation to the diagnostic possibility.

In the paediatric age group, it has been shown that the mechanism may be either hyperflexion or hyperextension, that the onset may be either immediate or delayed and that the lesion may be either complete or incomplete. The present state of knowledge does not permit the precise understanding of the micropathology, but it is felt that neither surgical nor pharmacological treatment can influence the extent of the paralysis or the possibility of recovery.

\section{ZUSAMMENFASSUNG}

Die Definition der paedriatischen Symptomatologie der traumatischen Myelopathie ohne sichtbare vertebrale Schädigung unterscheidet sich kaum von der Symptomatologie der mittleren und und älteren Altersgruppen. Obwohl in den späteren Altersgruppen der aetiologische Mechanismus besser verstanden ist, besteht doch eine erhebliche Unsicherheit bezüglich der Mikropathologie in beiden Altersgruppen, was zu Schwierigkeiten in der Erkennung, Diagnose und Behandlung führt.

In der paediatrischen Gruppe kann der Mechanismus entweder Hyperreflexion oder Hyperextension sein und die Schädigung kann entweder sofort oder verspätet erfolgen und kann entweder komplett oder inkomplett sein. Weder chirurgische noch pharmakologische Behandlung hat einen Einfluss auf die Ausdehnung der Lähmung noch auf die Möglichkeit der Wiederherstellung der Funktion.

\section{REFERENCES}

Ahmann, P. A., Smith, S. A., Schwartz, J. F. \& Clark, D. B. (1975). Spinal cord infarction due to minor trauma in children. Neurology, 25, 301-307.

Audic, B. \& MAURY, M. (I969). Secondary vertebral deformities in childhood and adolescence. Paraplegia, 7, IO-I6. 
BEDBROoK, G. M. (1966). Pathological principles in the management of spinal cord trauma. Paraplegia, 4, 43-56.

Brain, W. R., Northfield, D. W. C. \& Wilkinson, M. (I952). Neurological manifestations of cervical spondylosis. Brain, 75, 187-225.

Brain, Lord \& Wilkinson, M. (1967). Cervical Spondylosis. Heinemann, London.

BURKe, D. C. (I97I). Spinal cord trauma in children. Paraplegia, 9, I-I 2.

Burke, D. C. (1974). Traumatic spinal paralysis in children. Paraplegia, II, 268-276.

Byers, R. K. (1975). Spinal cord injuries during birth. Develop. Med. Child. Neurol. 17, IO3-IIO.

Crooks, F. \& Birkett, A. N. (1944). Fractures and dislocations of the cervical spine. Brit. F. Surg. 31, 252.

ForNI, I. (I947). Le fratture del rachide nel bambino. Chirurgia degli Organi de Movimento, 31, 347-36r.

Gelehrter, G. (1957). Die WirbelKörperbrüche in Kindes und Jugendalter. Archiv. für orthopädische und Unfall-Chirurgie, 49, 253-263.

Glasauer, F. E. \& Cares, H. L. (1972). Traumatic paraplegia in infancy. Fournal of the American Medical Association, 219, 38-4I.

Leventhal, H. R. (1960). Birth injuries of the spinal cord. F. Pediat. 56, 447-453.

MelZAK, J. (1969). Paraplegia among children. Lancet, 2, 45-48.

PARROT, J. (I869). Note sur un cas de rupture de la moelle chez un nouveau-ne par suit des manoeuvres pendant l'accouchement. Bulletin et Memoires de la Société de Médicine de Paris, 6, 38.

Schneider, R. C., Cherry, G. \& Pantek, H. (I954). The syndrome of acute central cervical spinal cord injury. F. Neurosurg. 2, 546-577.

TAYLOR, A. R. (I95I). The mechanism of injury to the spinal cord in the neck without damage to the vertebral column. F. Bone foint Surg. 33-B, 543-547.

WILKINSON, M. (I964). The morbid anatomy of cervical spondylosis and myelopathy. Brain, 83, 589-617.

Wolman, L. (1964). The neuropathology of traumatic paraplegia. Paraplegia, 1, 233-25r. 